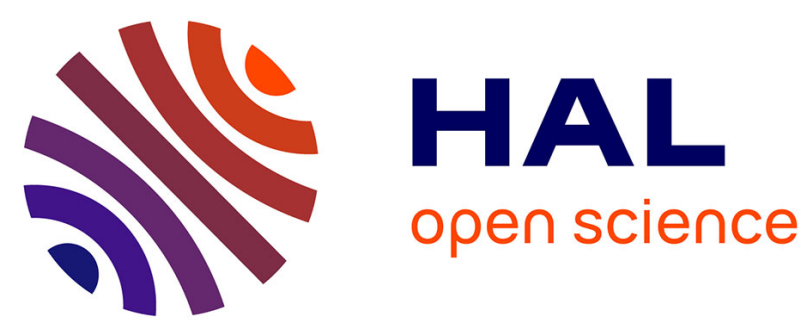

\title{
Cantilever-based microsystem for contact and non-contact deposition of picoliter biological samples
}

Pascal Belaubre, Matthieu Guirardel, Véronique Le Berre, Jean Bernard Pourciel, Christian Bergaud

\section{To cite this version:}

Pascal Belaubre, Matthieu Guirardel, Véronique Le Berre, Jean Bernard Pourciel, Christian Bergaud. Cantilever-based microsystem for contact and non-contact deposition of picoliter biological samples. Sensors and Actuators A: Physical , 2004, 110 (1-3), pp.130 - 135. 10.1016/j.sna.2003.09.024 . hal01849255

\section{HAL Id: hal-01849255 \\ https://hal.science/hal-01849255}

Submitted on 5 Jun 2019

HAL is a multi-disciplinary open access archive for the deposit and dissemination of scientific research documents, whether they are published or not. The documents may come from teaching and research institutions in France or abroad, or from public or private research centers.
L'archive ouverte pluridisciplinaire HAL, est destinée au dépôt et à la diffusion de documents scientifiques de niveau recherche, publiés ou non, émanant des établissements d'enseignement et de recherche français ou étrangers, des laboratoires publics ou privés. 


\title{
Cantilever-based microsystem for contact and non-contact deposition of picoliter biological samples
}

\author{
Pascal Belaubre $^{\mathrm{a}}$, Matthieu Guirardel ${ }^{\mathrm{a}}$, Véronique Leberre ${ }^{\mathrm{b}}$, \\ Jean-Bernard Pourciel ${ }^{\mathrm{c}}$, Christian Bergaud ${ }^{\mathrm{a}, *}$ \\ ${ }^{a}$ LAAS-CNRS, 7 Avenue du Colonel Roche, 31077 Toulouse Cedex 4, France \\ ${ }^{\mathrm{b}}$ Plateforme Transcriptome, Génopole de Toulouse Midi-Pyrénées, INSA/DGBA, 135 Avenue de Rangueil, 31077 Toulouse Cedex 4, France \\ ${ }^{c}$ LIMMS-CNRS/IIS, Institute of Industrial Science, The University of Tokyo, 4-6-4 Komaba, Meguro-ku, Tokyo 153-8505, Japan
}

Received 20 September 2002; received in revised form 20 August 2003; accepted 8 September 2003

\begin{abstract}
This paper describes a cantilever-based microsystem that permits the deposition of picoliter biological samples using a contact or non-contact method. Arrays of silicon-based cantilevers have been used to produce DNA microarrays. An electrowetting-on-dielectric (EWOD) principle is applied for the loading of the liquid by controlling surface tension. Deposition is achieved by direct contact between cantilevers and the surface by capillary transport. A non-contact deposition method has also been developed. It consists in an electric-field applied between the cantilevers and a conductive surface. The results obtained demonstrate that our system meets the need for producing high-density DNA, protein and cell chips.
\end{abstract}

(C) 2003 Elsevier B.V. All rights reserved.

Keywords: Biochips; Microcantilevers; Microarrays

\section{Introduction}

Biological differences in humans are due to genetic as well as environmental factors. These factors can be understood by studying variability in the genomic sequence and how each gene product is obtained in a given cell or tissue (gene expression).

By providing massive, parallel platforms for data gathering, microarray assays represent an essential technical advance in biomedical research. Biochips support advanced fabrication, detection and data mining technologies which in turn allow for data gathering at an unprecedented pace. They have revolutionized industrial microbiology and pathology research, while enabling fusion between genome research and drug discovery. Potential benefits of biochips are as follows:

- new detection methods, that will be more accurate and precise, can be developed,

- faster analytical processes are due to small dimensions,

- rapid gene expression monitoring and sequence analysis at the genomic level will therefore be allowed.

\footnotetext{
* Corresponding author.

E-mail address: bergaud@laas.fr (C. Bergaud).
}

Primary technologies used in microarray manufacture include photolithography, ink-jetting, mechanical microspotting and derivatives thereof. The photolithography approach relies on semiconductor technologies in a biochip setting. Photomasks direct spatially defined, solid phase DNA synthesis through the use of light which activates the modified phosphoramidite versions of the four DNA bases for DNA synthesis [1]. Piezoelectric technologies utilize versions of "ink-jet" printing to dispense sub-nanolitre volumes of reagents to defined locations. Electrically-active systems are used to deliver DNA bases, cDNAs, and other molecules via tiny delivery jets onto a solid surface [2]. An XYZ motion control system directs the jet location during this non-contact printing process. Microspotting technologies rely on direct surface contact. A printhead, moved by an $\mathrm{XYZ}$ motion control system, with microspotting pins, capillaries or tweezers allows transfer of pre-made substances from reagent trays onto solid surfaces [3,4].

Recently, the demand for massively parallel assay technology has spring up. Low cost and flexible techniques are, therefore, needed to meet specific requirements for increasingly integrated biochips. Also performance must be improved in terms of speed and sensitivity. To this end, promising approaches, mainly based on micro and nanotechnologies have been developed [5-8]. 
In this paper, a cantilever-based microsystem allowing deposition of picoliter biological samples with or without contact, is described. The originality of our work lies on the use of geometric dimensions, a minimal amount of biological samples, and the possibility of obtaining a great deal of depositions with a single loading process. Moreover, the simple mass production process of our cantilevers makes our system particularly cost effective.

\section{Fabrication process}

The technological process devised for microcantilevers fabrication is based on conventional microfabrication techniques (Fig. 1). The wafers used are $100 \mathrm{~mm}$ phosphorusdoped (100) silicon-on-insulator (SOI) substrate with a $5 \mu \mathrm{m}$-thick top Si layer. A low temperature silicon dioxide (LTO) is deposited by low pressure chemical vapor deposition (LPCVD) (Fig. 1A) and a lift-off process is carried out to define the aluminum electrodes (Fig. 1B). Electrode passivation is achieved by depositing a second low temperature silicon dioxide layer by LPCVD (Fig. 1C). The second part of the process consists in patterning and releasing the cantilevers by deep reactive ion etching (DRIE). Reactive ion etching (RIE) is then performed for silicon dioxide and a second etching is also carried out for the top Si layer to pattern the microcantilevers (Fig. 1D). During this step, microchannels and microreservoirs are etched through within the microcantilevers. Finally, a new DRIE is applied to the backside. Etching is stopped by the interfacial oxide layer of the SOI substrate. This silicon dioxide layer is then etched from the backside by RIE to release the cantilevers (Fig. 1E).

Arrays of cantilevers ( $2 \mathrm{~mm}$ long, $210 \mu \mathrm{m}$ wide, $5 \mu \mathrm{m}$ thick) with a spacing of $450 \mu \mathrm{m}$ were fabricated. Fig. 2 shows an array of two cantilevers with passivated aluminum electrodes located along channels and reservoirs. These

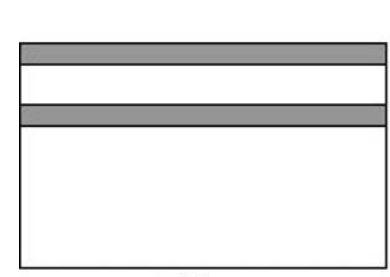

(A)

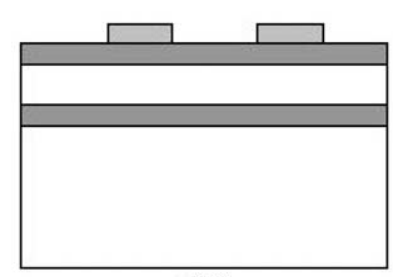

(B)

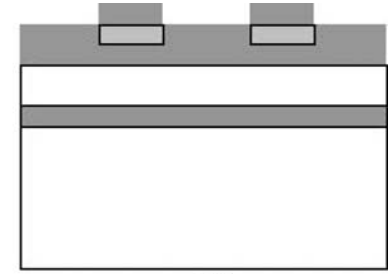

(C)

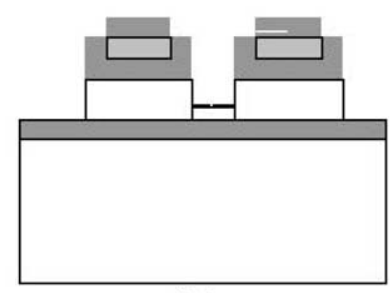

(D)

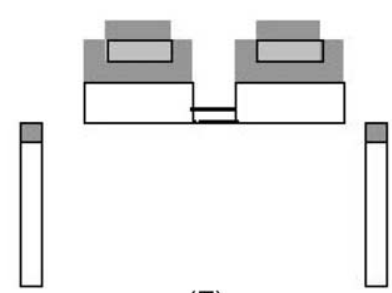

(E)

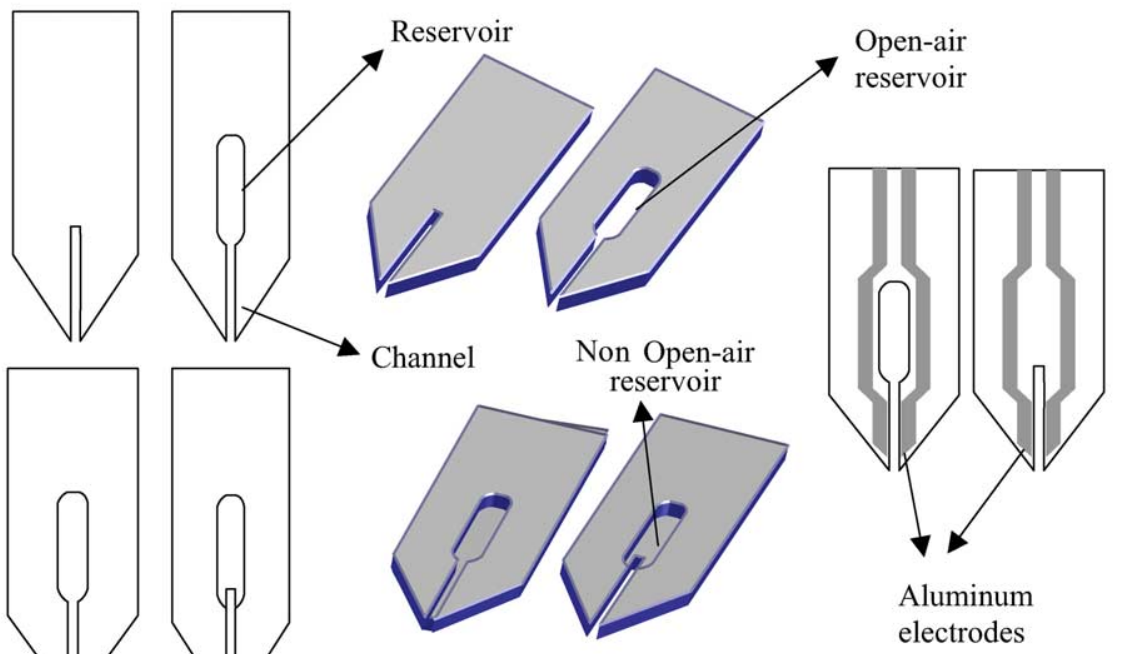

$(\mathrm{F})$

Fig. 1. Fabrication process of cantilevers (A-E) and resulting shapes (F). 


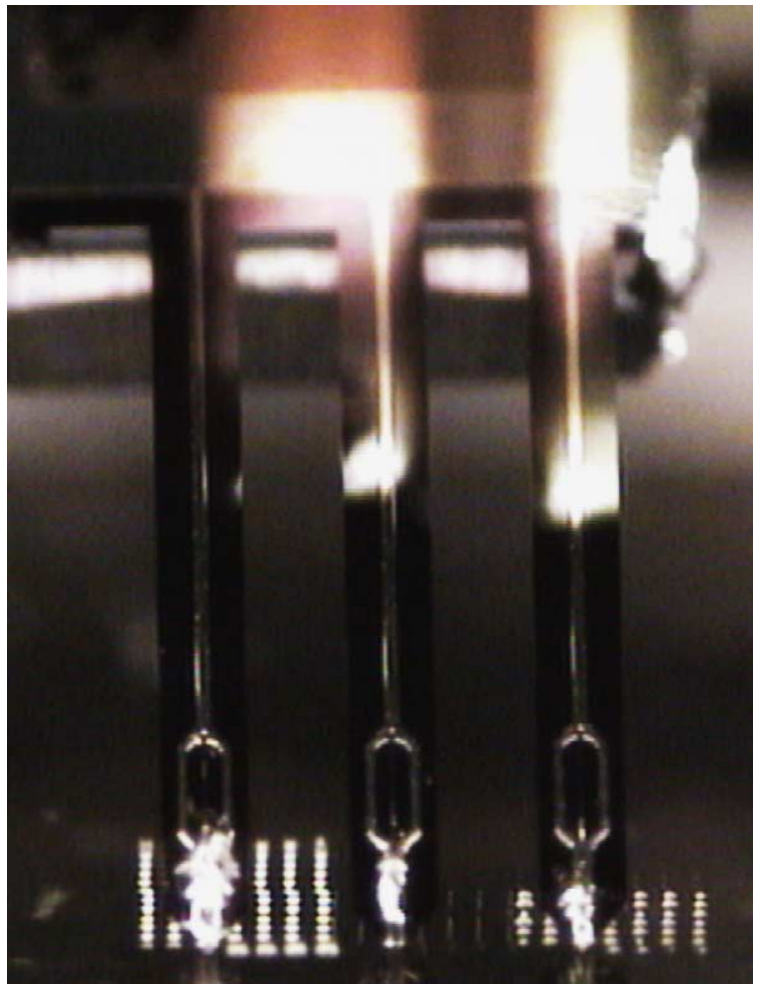

Fig. 2. Arrays of three cantilevers.

arrays are then used within the framework of a computercontrolled three-axis translation stage supplied by Sigma Koki that permits precise positioning of cantilevers relative to the surface as shown in Fig. 3a and b.

In the experimental procedure, an electrowetting-ondielectric principle is applied to improve the loading efficiency of the microchannels by electrically increasing the affinity of the liquid for the insulating layer. We developed this approach to compensate for the decreased surface wettability caused by the smaller microstructures even when using hydrophilic surfaces especially with high-aspect ratio narrow features such as microchannels.

\section{Experimental procedure}

\subsection{Electrowetting-on-dielectric principle: increasing the loading efficiency}

After dipping microcantilevers into the liquid to be deposited, an electric-field is applied between the passivated aluminum electrodes on the cantilevers and the conductive surface to increase the affinity of the liquid for the insulating layer. The electric-field results in a modified charge distribution that changes the free energy causing the liquid to spread and wet the surface of the cantilevers [9]. We used a low voltage electric-field (approximately $10 \mathrm{~V}$ during $10 \mathrm{~s}$ at $10 \mathrm{kHz}$ to avoid electrolysis), while the cantilevers were immersed in the biological sample, to load the reservoir. Even though the number of depositions made with one load process is very much dependent on the type of sample used, more than 50 depositions were carried out with almost all samples tested.

\subsection{Contact method for picoliter deposition}

As shown in Fig. 4, pre-wetted silicon-based microcantilevers have been used for depositing picoliter volumes of biological samples on a glass slide. Here, the method used consists in bringing the cantilevers in gentle contact with the glass surface.

Spots of a solution containing fluorescent labels (CY3) have also been deposited on a glass slide. Fig. 5a shows a fluorescence image obtained from a ScanArrayer ${ }^{\mathrm{TM}}$ instrument. Three cantilevers were used in parallel, one maintained in permanent contact to draw lines while the two others were intermittently in contact with the surface to create spots. Spots are approximately $10 \mu \mathrm{m}$ in diameter that is, almost ten times less than those achieved with a commercial instrument. Their oval shape results from the lack of control of the contact force that allowed the cantilevers to slip while in contact. Owing to its $10 \mu \mathrm{m}$ resolution, our ScanArrayer ${ }^{\mathrm{TM}}$ could hardly assess accurately these shapes. A scanner with a better resolution could be used to gain
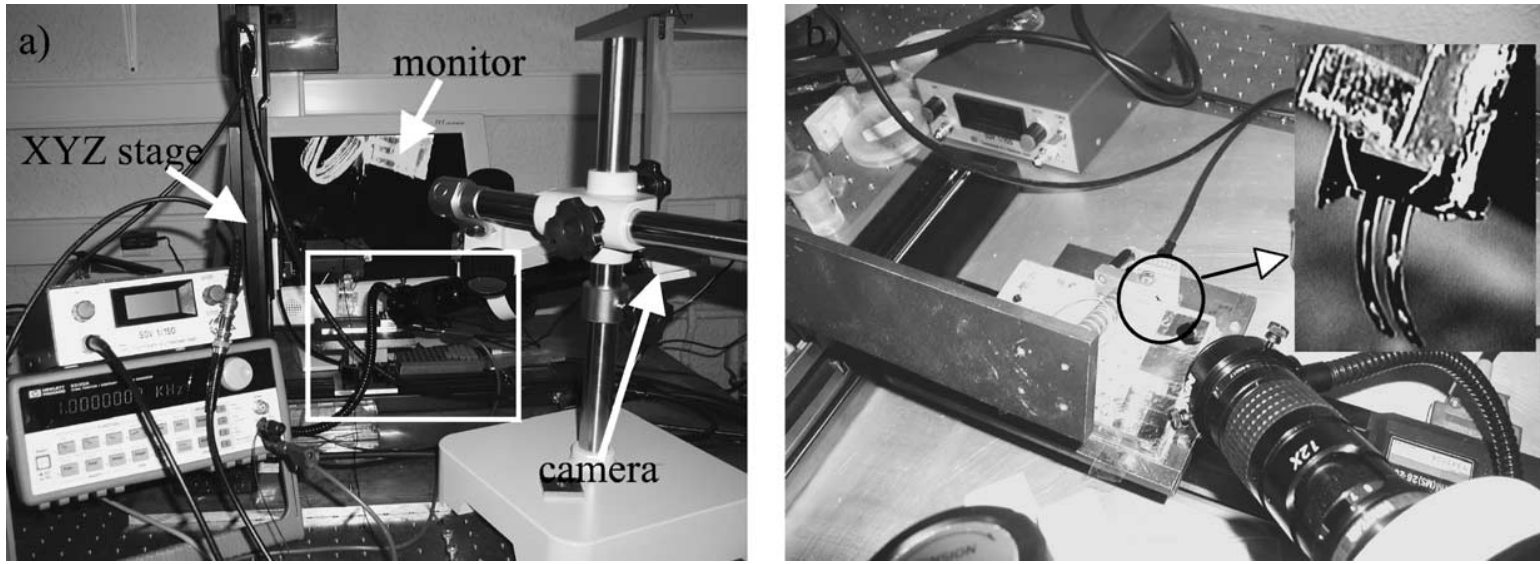

Fig. 3. XYZ motion control system with CCD camera monitoring (a) large view and (b) close-up view. 

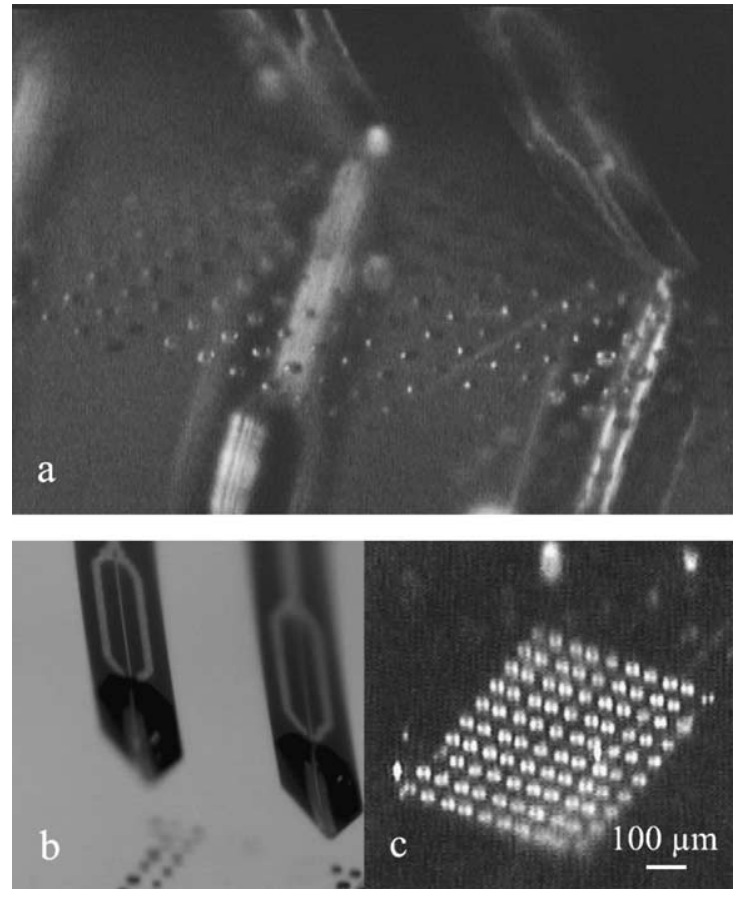

Fig. 4. (a) Two silicon-based microcantilevers during the deposition process; (b) pre-wetted silicon-based microcantilevers and (c) resulting spots after contacting the cantilevers with the surface of a glass slide.

better insights into the shapes of our spots. Fig. 5b shows an image acquired with BioAnalyzer Reader from LaVision Biotec, Bielefeld. The shape of our spots along with the effect of the cantilever slip leading to an oval shape instead of a circle one can easily be seen. Moreover we have been able to see that all our spots could be included in a circle of $10 \mu \mathrm{m}$ in diameter.

Since this technique relies on capillary transport, the shape of the spots depends on the surface wettability and on the angle made by the cantilever axis and the surface. This has been evidenced by hydrophilic and hydrophobic surfaces and by modifying this angle. The best results were obtained

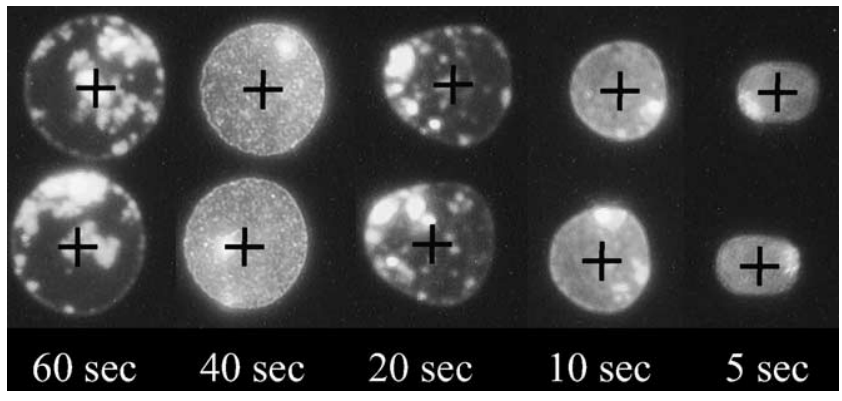

Fig. 6. Fluorescence microscope image of spots obtained by holding the cantilevers in contact with the surface for various time durations. It shows that both capillary transport and diffusion are involved in the spot formation. Spacing between each spot center is $70 \mu \mathrm{m}$.

with an angle of approximately $60^{\circ}$ using hydrophobic surfaces. The time during which the cantilevers are in contact with the surface also impacts the spot size as evidenced in Fig. 6 when comparing the diameter of spots achieved with 60, 40, 20, 10 and 5 s, respectively. As shown in Fig. 7, plotting the variation of the diameter spots as a function of the square root of the contact time gives a straight line. This $t^{1 / 2}$ dependence shows that diffusion also plays a major role in controlling the spot shape.

This shape is highly dependent on the amplitude of the force with which the cantilevers touch the surface. Indeed, for a spring constant of about $0.1 \mathrm{~N} / \mathrm{m}$, and a greater force contact, the cantilevers tend to bend (or even slip when the force is too high) thus enlarging the wetting surface between the cantilever end and the surface and, therefore, the amount of deposited liquid. In our case, this force was not precisely controlled. This could be achieved by using piezoresistive cantilevers to accurately monitor the contact force.

\subsection{Non-contact method for picoliter deposition}

Silicon-based cantilevers with non-passivated metallic electrodes have also been used to produce arrays of spots
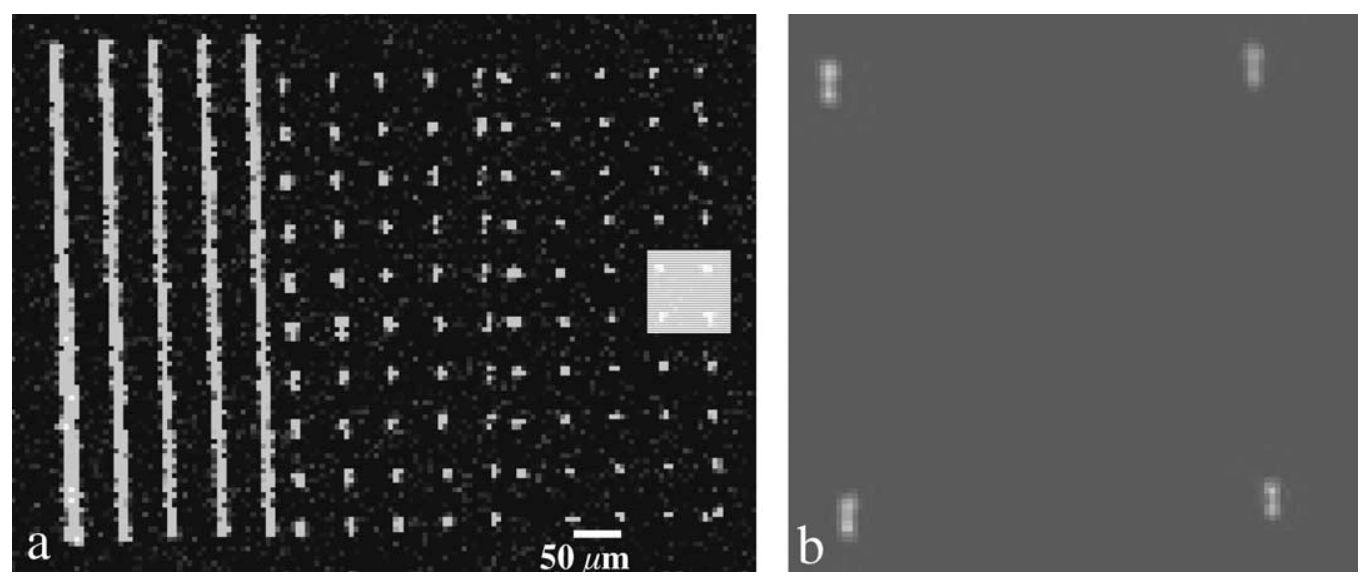

Fig. 5. Fluorescence image of lines and spots obtained from a solution containing fluorescent labels: (a) with a low resolution ScanArrayer ${ }^{\mathrm{TM}}$ and (b) with the BioTech BioAnalyser ${ }^{\mathrm{TM}}$. 


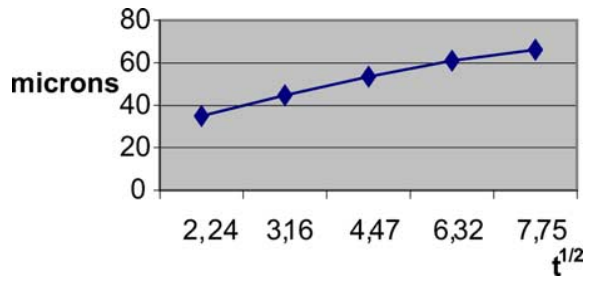

Fig. 7. Spot diameter variation vs. contact time duration.

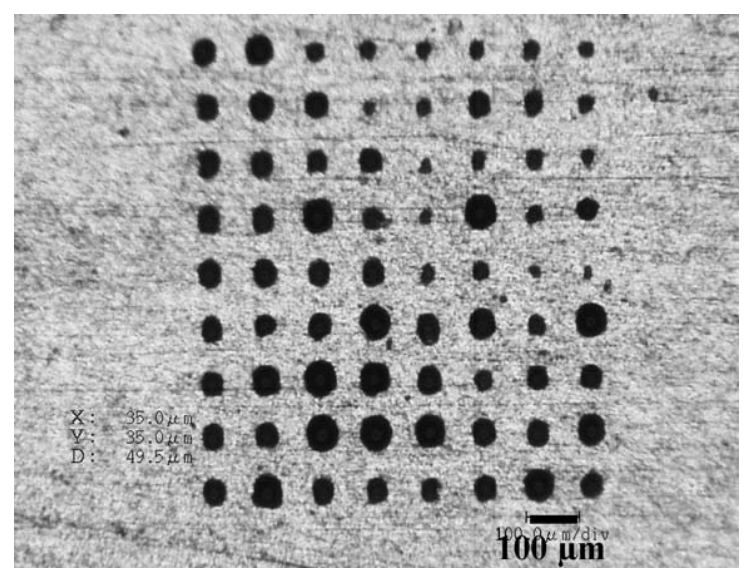

Fig. 8. Microarray of ink spots obtained using an electric-field-based technique for non-contact deposition. The diameter of the ejected spots varies from 10 to $50 \mu \mathrm{m}$.

by applying an electric-field between the electrodes and a conductive surface. This non-contact method was applied successfully to eject droplets of red ink on a copper surface. As can be seen in Fig. 8, the resulting spots are not uniform in shape and size. This can be accounted for by the fact that the surface was not perfectly smooth. This leads to non-uniformity in the applied field for a given voltage due to different a distance for each spot between the electrodes and the deposition surface. Shadow mask techniques can also be considered to achieve smaller and more uniform spots but this requires that the masks be perfectly flat and correctly positioned on the substrate. Current experiments aim at improving this deposition technique.

It should be pointed out that this non-contact method could be further improved to meet the requirements for use as an electrospray technique, provided that the operating conditions allow disintegration of the jet into charged droplets (ionization) [10].

\section{Conclusion and perspectives}

Silicon-based microcantilevers have been used for the deposition of picoliter biological samples. It has been demonstrated that this system can be used for contact and non-contact deposition. The results obtained demonstrate that our system perfectly matches the need for producing high-density DNA, protein as well as cell biochips.
In the near future, further improvements will be made on these cantilevers: piezoresistors will be integrated so that the contact process will be improved in terms of control of the contact force and the time during which the cantilevers touch the surface. Another important point concerns the optimization of the cantilever design to further reduce the volume of deposited biological samples. In this way the homogeneity of the spots will be increased and their size could be further decreased to reach the submicron level.

Tests still have to be realized on a biological aspect. Indeed, cross contamination is an important issue since deposition of two or more different biological samples with a single cantilever after a washing and drying process must be achieved.

\section{Acknowledgements}

This work was supported by The Genopole Toulouse Midi-Pyrénées.

\section{References}

[1] S.P.A. Fodor, J.L. Read, M.C. Pirrung, L. Stryer, A. Tsai Lu, D. Solas, Light-directed, spatially addressable parallel chemical synthesis, Science 251 (1991) 767.

[2] A. Blachard, Genetic Engineering, Principles and Methods, vol. 20, Plenum Press, New York, 1998.

[3] M. Schena, D. Shalon, R.W. Davis, P.O. Brown, Quantitative monitoring of gene expression patterns with a complementary DNA microarray, Science 270 (1995) 467.

[4] M. Schena, R.A. Heller, T.P. Theriault, K. Konrad, E. Lachenmeier, R.W. Davis, Microarrays: biotechnology's discovery platform for functional genomics, Trends Biotechnol. 16 (1998) 301.

[5] T. Laurell, L. Wallman, J. Nilson, Design and development of a silicon microfabricated flow-through dispenser for on-line picolitre sample handling, J. Micromech. Microeng. 9 (1999) 369-376.

[6] I. Papautsky, J. Brazzle, H. Swerdlow, A. Frazier, A low-temperature IC-compatible process for fabricating surface-micromachined metallic microchannels, J. Electromech. Systems 7 (2) (1998) 73, 267.

[7] R.S. Kane, S. Takayama, E. Ostuni, D.E. Ingber, G.M. Whitesides, Patterning proteins and cells using soft lithography, Biomaterials 20 (1999) 2363-2376.

[8] R.D. Piner, J. Zhu, F. Xu, S. Hong, C.A. Mirkin, "Dip-pen” nanolithography, Science 283 (1999) 661-663.

[9] J. Fowleer, T. Schoellhammer, J. Lee, H. Moon, C. Kim, Electrowetting and electrowetting-on-dielectric for microscale liquid handling, Sens. Actuators A 95 (2002) 256-268.

[10] V.N. Morozov, T.Y. Morozova, Electrospray deposition as a method for mass fabrication of mono- and multicomponent microarrays of biological and biologically active substances, Anal. Chem. 71 (15) (1999).

Biographies Pascal Belaubre was born in Aurillac in 1977. He received the engineer degree in electronics and informatics from the National Institute of Applied Sciences (INSA) in Toulouse in 2000 and the same year, a fifth year diploma (DEA) in automation. He is currently preparing the $\mathrm{PhD}$ degree in microelectronics and instrumentation at the Laboratory for Analysis and Architecture of Systems (LAAS-CNRS) in 
Toulouse, France. His research focuses on the development of deposition tools for the fabrication of DNA or proteins microarrays.

Matthieu Guirardel was born in Sarlat-la-Caneda in 1977. He received the ENSEA (Graduate School in Electronic and Electrical Engineering at Cergy, France) engineering degree in electronics in 2000 and the same year, a fifth year diploma (DEA) in electronic and instrumentation from University Paris VI. Currently, he is preparing for the $\mathrm{PhD}$ degree in electronics at the Laboratory for Analysis and Architecture of Systems-CNRS, Toulouse, France. His research involves the development of microelectromechanical biosensors.

Véronique Leberre was born in Paris in 1970. She received the PhD degree in 1997 from the Pharmacology and Structural Biological Institute in Toulouse (France). She has worked on the extraction and purification of a protein inhibitor of the amylase. From 1997 to 1999, she was a post-doctoral fellow at the National Institute of Applied Sciences in Toulouse where she investigated the cell wall rescue mechanism in the yeast Saccharomyces cerevisiae, using molecular biology technologies. Currently, she is a CNRS researcher at the Transcriptom-Biopuces Platform from the MidiPyrénées, Region's Genopole. Her research involves the development of a new generation of biochips using the 'dendrimeric' technology for fixation of the nucleic acids and she is involved in the development of novel approaches to design micro and nanosystems for DNA chip applications.
Dr. Jean-Bernard Pourciel was born in Toulouse (France) in April 1947. He received his $\mathrm{PhD}$ degree in April 1974, in France at Laboratoire d'Analyse et d'Architecture des Systèmes (LAAS) of CNRS till 1999 in the field of computer process control, he is now a senior visiting researcher in Japan at the LIMMS/CNRS (Laboratory for Integrated Micro Mechatronics Systems), a joint laboratory dedicated to MEMS between the French CNRS and the University of Tokyo. His concern is to use cantilever-based methods and original computer control algorithms for micro-metrology for MEMS, particularly for measuring profiles into deep electro-discharge machined microholes. He is also involved in the study of silicon-based systems to deposit picoliter of liquids (in collaboration with LAAS/CNRS).

Christian Bergaud was born in Domont (France) in 1968. He received the engineer degree in physics from the National Institute of Applied Sciences in Lyon (France) in 1991 and the PhD degree in electronics from the National Institute of Applied Sciences in Toulouse (France) in 1994. Between 1995 and 1996, he was a post-doctoral fellow at the University of Tokyo where he studied the development of microsystems dedicated to metrological applications. Currently, he is a CNRS researcher at the Laboratory for Analysis and Architecture of Systems (LAAS) at Toulouse. His research involves physical characterization of micro and nanostructures and the development of novel approaches to design micro and nanosystems for biological applications. 